Citation: Scott E.C. et al. (2018) Autologous Stem Cell Transplant in the Era of Bortezomib-Based Induction for AL Amyloidosis - A Single Institution 11 Year Experience. Science Publishing Group Journal 1(2).

Corresponding Author:

Emma C. Scott, MD

Associate Professor of Medicine Division of Hematology \& Oncology Oregon Health \& Science University 3181 SW Sam Jackson Park Road, UHN 73C, Portland, OR 97239 Phone: (503) 494-8534

Fax: (503) 494-3257

E-mail: scottem@ohsu.edu

Thesis data were presented in part at the 2017 American Society of Hematology National Meeting.

Disclaimers: None

Copyright:

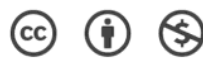

(C) 2018 Science Publishing Group

This open access article is distributed under the terms of the Creative

Commons Attribution Non-

Commercial License.
REVIEW ARTICLE

\section{Autologous Stem Cell Transplant in the Era of Bortezomib-Based Induction for AL Amyloidosis - A Single Institution 11 Year Experience}

\author{
Carlton D. Scharman, MD ${ }^{1}$, Richard T. Maziarz, MD², \\ Joseph J. Shatzel, $\mathrm{MD}^{2}$, Eva Medvedova, $\mathrm{MD}^{2}$, Alex Stentz, $\mathrm{MPH}^{2}$, \\ Gloria Rhyne, $\mathrm{MA}^{2}$, Chafic Karam, $\mathrm{MD}^{3}$, Rupali S. Avasare, $\mathrm{MD}^{4}$, \\ Stephen B. Heitner, MD ${ }^{5}$, Emma C. Scott, MD $^{2}$ \\ Author's affiliations: \\ 1. Department of Internal Medicine \\ 2. Center for Hematologic Malignancies, Knight Cancer Institute \\ 3. Department of Neurology \\ 4. Division of Nephrology \& Hypertension \\ 5. Knight Cardiovascular Institute, Oregon Health \& Science University, \\ Portland. Oregon
}

\section{Abstract:}

A single-institution retrospective outcome analysis was performed on a cohort of 52 patients with systemic light-chain amyloidosis comparing bortezomib-based induction versus no induction before autologous stem cell transplantation (ASCT). Thirteen patients underwent ASCT without induction, 33 received bortezomib-based induction, and 6 received non-bortezomib-based induction. Thirty patients received melphalan $200 \mathrm{mg} / \mathrm{m}^{2}$, while 22 received dose-reduced melphalan. Treatment-related mortality at 100 -days was $3.8 \%$. Overall hematologic (ORR) and organ response rates (OR) in the entire cohort were $85 \%$ and $67 \%$, respectively. ORR was $94 \%$ in those receiving bortezomib-based induction versus $69 \%$ with no induction $(p=0.04)$. Median time to maximum hematologic response post ASCT was significantly shorter with bortezomib-based induction (3 months versus 14 months). Five-year progression-free survival (PFS) and overall survival (OS) in the entire cohort were $50 \%$ and $73 \%$, respectively. Between groups there were no statistically significant differences in depth of hematologic response, cardiac or renal response rate, or 5-year PFS or OS. This study supports ASCT, with bortezomib-based induction as a well-tolerated therapy, with higher ORR and shorter time to maximum hematologic response than no induction. Larger-scale multi-center randomized trials would be beneficial to confirm these observations. 


\section{Introduction:}

Systemic immunoglobulin light chain (AL) amyloidosis is a monoclonal plasma cell disorder characterized by the deposition of insoluble light (AL) or heavy-chain (AH) fragments into tissues, often resulting in progressive organ dysfunction if left untreated. Although novel agents directly targeting amyloid protein are currently being studied, treatment has historically been directed at suppressing bone marrow plasma cell growth given that these are the source of the pathologic protein.

With few exceptions, patients with AL amyloidosis require systemic treatment at the time of diagnosis including consideration of autologous stem cell transplantation (ASCT), for which eligibility criteria are largely country- and institution-dependent. ${ }^{1}$ The combination of high-dose melphalan (HDM) conditioning with ASCT for selected patients has shown considerable success, with upfront ASCT without induction carrying hematologic responses ranging from 32-66\% and organ responses ranging from $33-78 \%{ }^{2-7}$ Deeper hematologic responses have been associated with longer survival. ${ }^{2,8}$

Outcomes of ASCT have improved over time, likely due to advancements and peri-transplant supportive care, as well as improved center experience in patient selection and in managing those with complex medical conditions. Notably, a large retrospective analysis of the Center for International Blood and Marrow Transplant Research (CIBMTR) database, which assessed outcomes of AL amyloidosis patients treated in various time periods, has shown that early posttransplantation mortality and survival have improved in recent years (100-day mortality of 5\% and 5-year overall survival of $77 \%$ for patients treated from 2007-2012, compared to 100-day mortality of $11 \%$ and 5 -year overall survival of $61 \%$ in 2001-2006). ${ }^{1}$ However, patients with cardiac amyloidosis did not share these similar improvements.

Currently, there are no agents approved by the Food and Drug Administration (FDA) or European Medicines Agency (EMA) for induction prior to ASCT in patients with AL amyloidosis. It therefore remains largely institution-dependent regarding decision-making on proceeding directly to ASCT without induction versus use of front-line therapy (e.g., chemotherapy, immunomodulatory agents, proteasome inhibitors) in an off-label manner for the purposes of improving organ function prior to transplant. One agent that has received considerable attention in this setting is bortezomib, a proteasome inhibitor currently FDA-approved for treatment of multiple myeloma. ${ }^{9}$ Though most efficacy data are largely limited to case series, bortezomib appears to be active in patients with AL amyloidosis, and has received consideration in the setting of induction, consolidation, and as stand-alone therapy for those ineligible for transplant or with relapsed disease. ${ }^{10}$

Our group previously published a retrospective analysis of 31 patients comparing bortezomibbased induction versus no induction prior to ASCT. ${ }^{11}$ This preliminary analysis showed that compared to no induction, bortezomib-based induction was associated with higher hematologic, organ, and cardiac response rates with shorter time to maximum hematologic response (though testing for statistical significance was not performed due to sample size). Here we report an update, with an increase in sample size to 52 patients and with a long-term follow-up extending to 11 years. 


\section{Subjects and Methods:}

This study was designed as a retrospective cohort analysis that was conducted at a single tertiary care academic medical center (Oregon Health \& Science University). Prior to data collection, this study received institutional review board approval. All patients with biopsy-confirmed systemic immunoglobulin AL amyloidosis who received HDM + ASCT at Oregon Health \& Science University (OHSU) from 2006 to 2017 were included $(n=52)$.

All patients had a detectable monoclonal protein in the serum and/or urine. Amyloidosis was detected on organ or bone marrow biopsy specimens by positive Congo red or toluidine blue staining, and then confirmed histologically via immunohistochemical staining or mass spectroscopy (Mayo Clinic Laboratories, $2001^{\text {st }}$ Street SW, Rochester, MN 55905).

Institutional eligibility criteria for performing HDM + ASCT for AL amyloidosis patients included: biopsy-proven AL amyloid, Eastern Cooperative Oncology Group (ECOG) performance status $\leq 2$, left ventricular ejection fraction $\geq 45 \%$, New York Heart Association class I or II, Mayo clinic cardiac biomarker criteria stage I or II, carbon monoxide diffusing capacity $>50 \%$, and serum creatinine $\leq 3.0 \mathrm{mg} / \mathrm{dL}$ unless receiving dialysis. There was no upper age limit.

Patients received melphalan $200 \mathrm{mg} / \mathrm{m}^{2}$ or dose-reduced melphalan $\left(100-140 \mathrm{mg} / \mathrm{m}^{2}\right)$ for higherrisk patients: $140 \mathrm{mg} / \mathrm{m}^{2}$ was provided for those $\geq 70$ years of age, NYHA class $\geq 11$ heart failure, serum creatinine $>2.0 \mathrm{mg} / \mathrm{dL}$ or dialysis dependence, or ECOG score of $2 ; 100 \mathrm{mg} / \mathrm{m}^{2}$ was provided for those with NYHA class III heart failure. HDM was administered over 2 days (day -2 and 1 ) in divided dose in the setting of oral cryotherapy. Peripheral blood stem cells were then mobilized with granulocyte colony-stimulating factor (G-CSF) while monitoring for toxicity. If there was predicted lower stem cell yield with G-CSF alone, plerixafor was added based on institutional protocol. $^{12}$

Prior to ASCT, those receiving bortezomib induction could have received one of several bortezomib-containing regimens, including (but not limited to) bortezomib with dexamethasone (VD), bortezomib with lenalidomide and dexamethasone (RVD), or bortezomib with cyclophosphamide and dexamethasone (CyBorD). VD treatment schedules were: IV bortezomib $1.3 \mathrm{mg} / \mathrm{m}^{2}$ on days $1,4,8$, and 11 or days 1,8 , and 15 with oral dexamethasone 20 to $40 \mathrm{mg}$ on each day of bortezomib on 21-day cycles; or subcutaneous bortezomib $1.5 \mathrm{mg} / \mathrm{m}^{2}$ on days 1,8 , and 15 with oral dexamethasone 20 to $40 \mathrm{mg}$ on each day of bortezomib on 28-day cycles. ${ }^{13}$ The RVD treatment schedule was lenalidomide 15 to $25 \mathrm{mg}$ orally on days $1-21$; bortezomib 1.3 to $1.5 \mathrm{mg} / \mathrm{m}^{2}$ on days $1,8,15,22$, with oral dexamethasone 20 to $40 \mathrm{mg}$ with each dose of bortezomib. The CyBorD treatment schedule was subcutaneous bortezomib $1.5 \mathrm{mg} / \mathrm{m}^{2}$, oral cyclophosphamide 300 $\mathrm{mg} / \mathrm{m}^{2}$, oral dexamethasone $40 \mathrm{mg}$ on days 1,8 , and 15 on 28 -day cycles. ${ }^{14}$ Assessment of hematologic and organ involvement, response, and progression were based on consensus criteria reported in the $10^{\text {th }}$ International Symposium on Amyloid and Amyloidosis and the updated criteria from the Roundtable on Clinical Research in Immunoglobulin Light-Chain Amyloidosis. ${ }^{15-17}$ Complete hematologic response (CR) was defined as normalization of the free light chains levels and ratio, with negative serum and urine immunofixation. Very good partial response (VGPR) was defined as a reduction in the difference between involved and uninvolved free light chain (dFLC) to $<40 \mathrm{mg} / \mathrm{L}$. Partial response (PR) was defined as a greater than $50 \%$ reduction in the dFLC. No 
response (NR) was defined as less than a PR but without progressive disease. Organ response was evaluated on the basis of improvement of one or more affected organs. Only one parameter was required to satisfy the organ response criteria, and the response needed to be maintained for a minimum of 3 months to be considered valid. Renal response required a $30 \%$ reduction in 24-hour urine protein excretion (at least $0.5 \mathrm{~g} / \mathrm{d}$ ) with stable creatinine. Cardiac response required one of (1) improvement by 1 NYHA classes if baseline class 2 or 2 NYHA classes if baseline Icass 3 or 4 without an increase in diuretic use; (2) NT-proBNP improvement by $>30 \%$ and $>300$ $\mathrm{ng} / \mathrm{L}$ decrease if baseline NT-proBNP level $\geq 650 \mathrm{ng} / \mathrm{L}$. Hepatic response required either (1) $50 \%$ decrease in (or normalization of) an initially elevated alkaline phosphatase level or (2) reduction in the size of the liver by at least $2 \mathrm{~cm}$ by radiographic determination. A neurologic response was defined as either a reduction in the Neuropathy Impairment Score (NIS) by 10 points, Gastrointestinal tract improvement was defined as reduction of diarrhea to $50 \%$ of previous movements per day, or decrease in fecal fat excretion by $50 \%$.

\section{Statistical Analysis}

Data analysis was performed comparing patients who received a bortezomib-containing induction regimen versus no induction prior to ASCT. Categorical data for both groups were analyzed by using a two-tailed Fisher exact test. A Student $t$ test was used for continuous data. Hematologic response rate (ORR) was defined as the percentage of patients having a partial hematologic response or greater. Organ response rate (OR) was defined as the percentage of patients who had a response of any involved organ per consensus criteria. Progression-free survival (PFS) was defined as the time from day 0 of ASCT to the date of relapse or progression of disease or death from any cause; observation was censored at the date of last follow-up for patients alive at last contact. Overall survival (OS) was defined as the time from day 0 of ASCT to death from any cause; observation was censored at the date of last follow-up for patients alive without relapse/progression of disease at last contact. These were estimated using Kaplan-Meier analysis. Survival curves were compared using log-rank analysis. Prism 3.02 statistical software was used for statistical analysis.

\section{Results:}

\section{Clinical Characteristics}

Baseline characteristics, including disease features and organ involvement, are shown in Table 1. A total of 52 patients with systemic AL amyloidosis who underwent ASCT were identified between January 1, 2005 and December 31, 2017. Thirty-two patients underwent ASCT in the recent years of 2005-2012, the time period of our original study. Twenty additional patients were treated in the years of 2013-2017. Median patient age at the time of transplant was 61.5 years (range 41-72 years). Renal involvement was the most common organ involved (31 patients, 59\%), followed by cardiac ( 23 patients, $43 \%$ ), and peripheral nerve ( 9 patients, $17 \%$ ). Thirty-one patients $(60 \%)$ had involvement of multiple organs; 14 patients $(27 \%)$ had involvement of three or more organs. Thirty patients (58\%) received a melphalan dose of $200 \mathrm{mg} / \mathrm{m}^{2}$ prior to ASCT, whereas 22 patients $(42 \%)$ required dose-reduction. 
Table 1. Baseline characteristics of 52 patients with AL amyloidosis before autologous stem cell transplantation

\begin{tabular}{|l|c|}
\hline \multicolumn{1}{|c|}{ Characteristic } & $\underline{\text { Value }}$ \\
\hline Age at Transplant, Mean Years (SD) & $61.0(7.2)$ \\
\hline Sex, $\mathbf{n}(\%)$ & \\
Male & $31(58)$ \\
Female & $22(42)$ \\
\hline Hematopoietic Cell Transplant Co-Morbidity & $3.1(2.2)$ \\
Index, Mean Score (SD) & \\
\hline Karnofsky Performance Status, Mean Score (SD) & $83.3(9.0)$ \\
\hline Light Chain Type, $\mathbf{n}(\%)$ & \\
Kappa light chain & $15(29)$ \\
Lambda light chain & $37(71)$ \\
\hline Organ Involvement, $\mathbf{n}$ (\%) & \\
Single organ & $21(40)$ \\
Two organs & $17(33)$ \\
Three or more organs & $14(27)$ \\
Cardiac & $23(43)$ \\
Renal & $31(59)$ \\
Gastrointestinal & $11(21)$ \\
Peripheral neuropathy & $9(17)$ \\
Liver & $8(15)$ \\
Autonomic neuropathy & $5(09)$ \\
Skin & $3(06)$ \\
Macroglossia & $2(04)$ \\
\hline Melphalan Dose Received, $\mathbf{n}$ (\%) & $30(58)$ \\
200 mg/m ${ }^{2}$ & $18(34)$ \\
140 mg/m ${ }^{2}$ & $4(08)$ \\
100 mg/m ${ }^{2}$ & \\
\hline
\end{tabular}

Of the 52 total patients, 13 proceeded directly to ASCT after diagnosis without induction, whereas 33 received a bortezomib-based induction regimen (increased from 12 in our previous study, reflecting our institutional commitment to change of practice), and 6 received a nonbortezomib based form of induction treatment. All patients in the newer cohort (i.e., treated from 2013 to 2017) received bortezomib induction. In total, 18 patients received CyBorD, 15 patients received VD, and 9 patients received RVD.

Patients initially transplant ineligible due to advanced cardiac involvement or poor performance status

At the time of diagnosis, pre bortezomib induction, 4 subjects were Mayo clinic cardiac stage III at diagnosis, and improved to stage II, 2 subjects were NYHA stage III (2) improved to stage I (1) 
and II (2) and a further 3 subjects had an ECOG PS of 3 at diagnosis, improved to 2 (2) and 1 (1) rendering them transplant eligible. Overall 9 out of 33 subjects, deemed transplant ineligible prior to bortezomib induction (\%) became eligible for transplant due to improvement in these factors.

\section{Hematologic and Organ Responses}

The overall hematologic (ORR) and organ response rates (OR) at 1-year post-ASCT in the entire cohort of 52 patients were $85 \%$ and $67 \%$, respectively (Table 2). Forty percent of patients achieved a CR, 27\% VGPR, 15\% PR, 6\% stable disease (SD), and $8 \%$ progressive disease (PD). While all patients in the newer cohort received bortezomib-containing induction regimens, response rates in the early patient cohort (2005-2012) were largely comparable to those managed more recently (2013-2017).

Table 2. Hematologic (ORR) and organ response (OR) rates. Not shown are 6 patients who received non-bortezomib-based induction. $C R=$ complete response; VGPR = very good partial response; $P R=$ partial response; $S D=$ stable disease; $P D=$ progressive disease

\begin{tabular}{|c|c|c|c|c|}
\hline & $\begin{array}{l}\text { Total Co- } \\
\text { hort, n } \\
(\%)\end{array}$ & $\begin{array}{l}\text { Bortezomib } \\
\text { Induction, } \\
\text { n (\%) }\end{array}$ & $\begin{array}{l}\text { No- } \\
\text { Induction, } \\
\text { n (\%) }\end{array}$ & $\begin{array}{l}\text { P. } \\
\text { Value }\end{array}$ \\
\hline $\begin{array}{l}2005-2012 \\
\text { Cohort }\end{array}$ & $N=32$ & $N=13$ & $N=13$ & \\
\hline ORR & $25(78)$ & $12(92)$ & $9(69)$ & \\
\hline CR & $14(44)$ & $5 \quad(38)$ & $6(46)$ & \\
\hline VGPR & 6 (19) & $4 \quad(31)$ & $2(15)$ & \\
\hline PR & 5 (16) & 3 (23) & $1(08)$ & \\
\hline SD & $2(06)$ & $1 \quad(08)$ & 0 & \\
\hline PD & 4 (13) & 0 & $3(23)$ & \\
\hline Unknown & 1 & 0 & 1 & \\
\hline $\begin{array}{l}\text { 2013-2017 } \\
\text { Cohort }\end{array}$ & $N=20$ & $N=20$ & & \\
\hline ORR & $19(95)$ & $19(95)$ & $n / a$ & \\
\hline CR & $7 \quad(35)$ & $7 \quad$ (35) & $\mathrm{n} / \mathrm{a}$ & \\
\hline VGPR & $9 \quad(45)$ & $9 \quad(45)$ & $n / a$ & \\
\hline PR & 3 (15) & 3 (15) & $\mathrm{n} / \mathrm{a}$ & \\
\hline SD & 1 (05) & 1 (05) & $\mathrm{n} / \mathrm{a}$ & \\
\hline PD & 0 & 0 & $\mathrm{n} / \mathrm{a}$ & \\
\hline Total Cohort & $N=52$ & $N=33$ & $N=13$ & \\
\hline ORR & $44(85)$ & $31(94)$ & $9(69)$ & 0.04 \\
\hline
\end{tabular}




\begin{tabular}{|l|l|l|l|l|}
\hline CR & $21(40)$ & $12(36)$ & $6(46)$ & \\
\hline VGPR & $14(27)$ & $13(39)$ & $2(15)$ & \\
\hline PR & $8(15)$ & $6(18)$ & $1(08)$ & \\
\hline SD & $3(06)$ & $2(06)$ & 0 & \\
\hline PD & $4(08)$ & 0 & $3(23)$ & \\
\hline \multicolumn{7}{|l|}{} \\
\hline OR & $33 / 49(67)$ & $23 / 30(77)$ & $7 / 13(54)$ & 0.16 \\
\hline Cardiac & $13 / 21(62)$ & $10 / 14(71)$ & $3 / 7(43)$ & 0.24 \\
\hline Renal & $18 / 27(67)$ & $13 / 18(72)$ & $5 / 9(56)$ & 0.68 \\
\hline
\end{tabular}

ORR at 1-year post-ASCT was $94 \%$ and $69 \%$, respectively ( $p=0.04$ in patients who received bortezomib induction vs. no induction). In the 33 patients who received bortezomib-containing induction, ORR was $85 \%$ prior to ASCT, and increased to $94 \%$ post-ASCT. The median time to maximum hematologic response post -ASCT was significantly shorter with bortezomib induction compared to no induction (3 months vs. 14 months). Twenty-five (76\%) patients receiving bortezomib induction had a response of VGPR or CR post-transplant compared to $8(62 \%)$ patients receiving no-induction $(\mathrm{p}=0.46)$.

In the entire cohort, organ-specific responses included 13 cardiac, 18 renal, 4 gastrointestinal, 4 hepatic, and 3 neurologic. OR was $77 \%$ in the group that received bortezomib induction compared to $54 \%$ in those with no induction $(p=0.16)$. The cardiac response rate for the entire cohort was $62.0 \%$, with a rate of $71 \%$ in those pre-treated with bortezomib versus $43 \%$ in those who did not receive induction therapy $(p=0.24)$. Renal response rates were $72 \%$ and $56 \%$ in those receiving bortezomib induction vs. no induction, respectively $(p=0.68)$.

\section{Early (Day 100) Mortality}

Two patients died within 100 days after ASCT (3.8\% of the overall study population), all occurring in the 2005-2012 cohort in those who received no induction. One patient died early postASCT due to exsanguination secondary to spontaneous rupture of an AL-involved spleen. The second patient, who had cardiac involvement, died due to complications of congestive heart failure. No mortalities occurred during stem cell mobilization.

\section{Survival}

With a median follow-up of 2.92 years, 5-year PFS and OS in the entire cohort were $50 \%$ and $73 \%$, respectively (Figure 1). In those with cardiac involvement, these rates were $59 \%$ and $83 \%$, respectively. The median PFS time for the entire study population was 5.8 years, whereas the median OS has not yet been reached. 


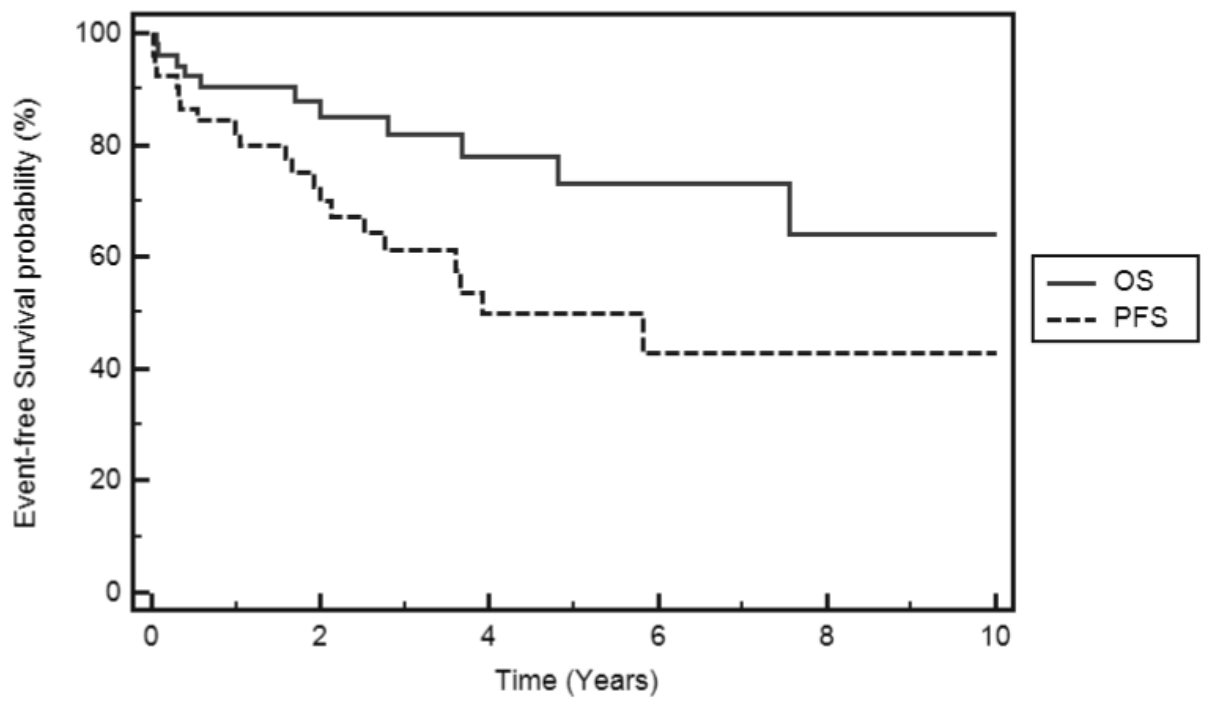

Figure 1. Overall survival (OS) and progression-free survival (PFS) in the total study population

$$
(n=52) \text {. }
$$

One-, 5-, and 10-year OS for the bortezomib group were $96.9 \%, 85.9 \%$, and $85.9 \%$ respectively (Figure 2), compared to $76.9 \%$ ( $p=0.03$ compared to bortezomib at 1 -year), 59.8\% ( $p=0.11)$, and $59.8 \%(p=0.11)$, respectively. Comparing bortezomib-based induction versus no induction (Figure 3), PFS at 1 -year was $87.0 \%$ and $69.2 \%$, respectively $(p=0.06)$, and at both 5 -years and 10 years was $58.8 \%$ and $43.3 \%$, respectively $(p=0.57 \%)$. In those with cardiac involvement, 5 -year OS in the bortezomib-induction group was $86 \%$ compared to $50 \%$ in those who received no induction ( $p=0.17$, Figure 4 ); 5 -year PFS was $50.6 \%$ compared to $37.5 \%$, respectively ( $p=0.61$, Figure 5). For all survival outcomes assessed, 5 -year survival rates (and therefore $p$-values) were identical to those at the 10 -year timepoint.

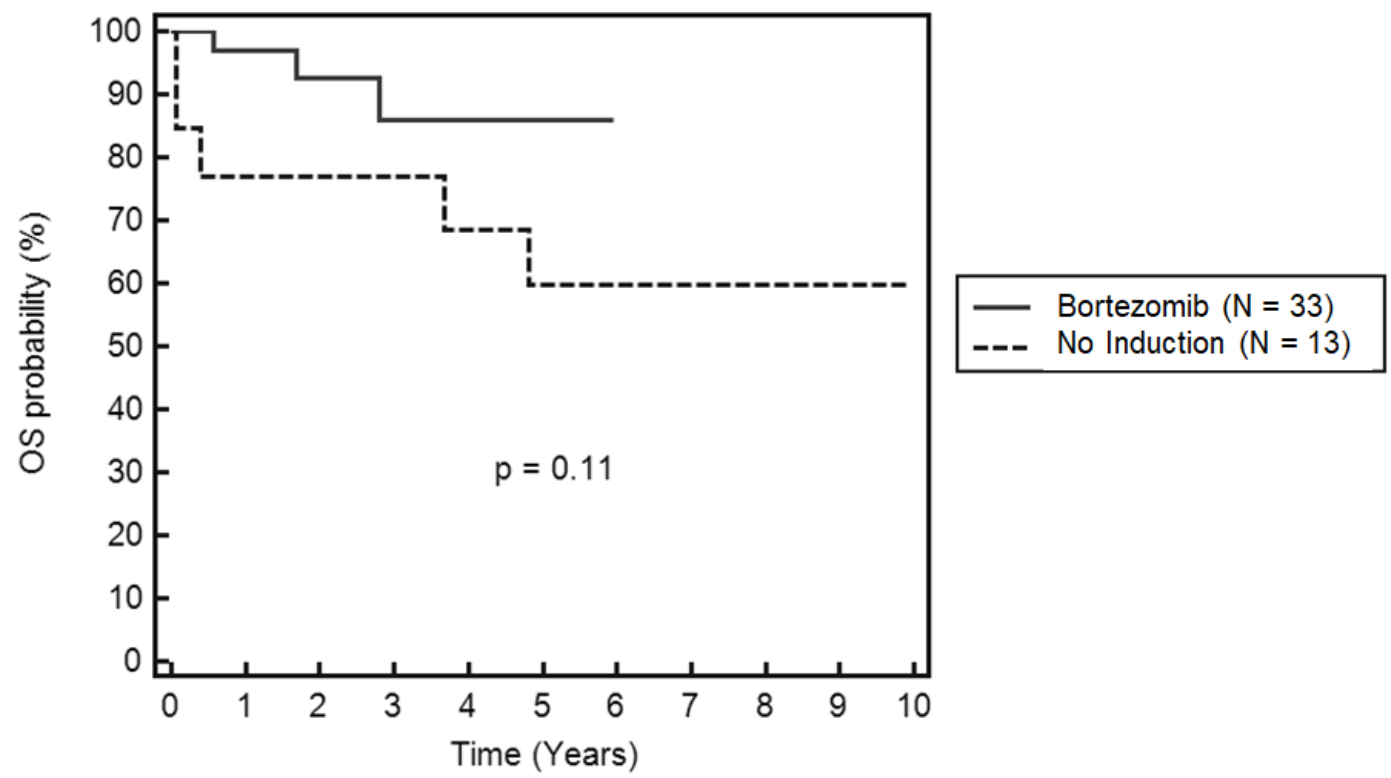

Figure 2. Overall survival (OS) in those receiving bortezomib induction compared to no induction. 


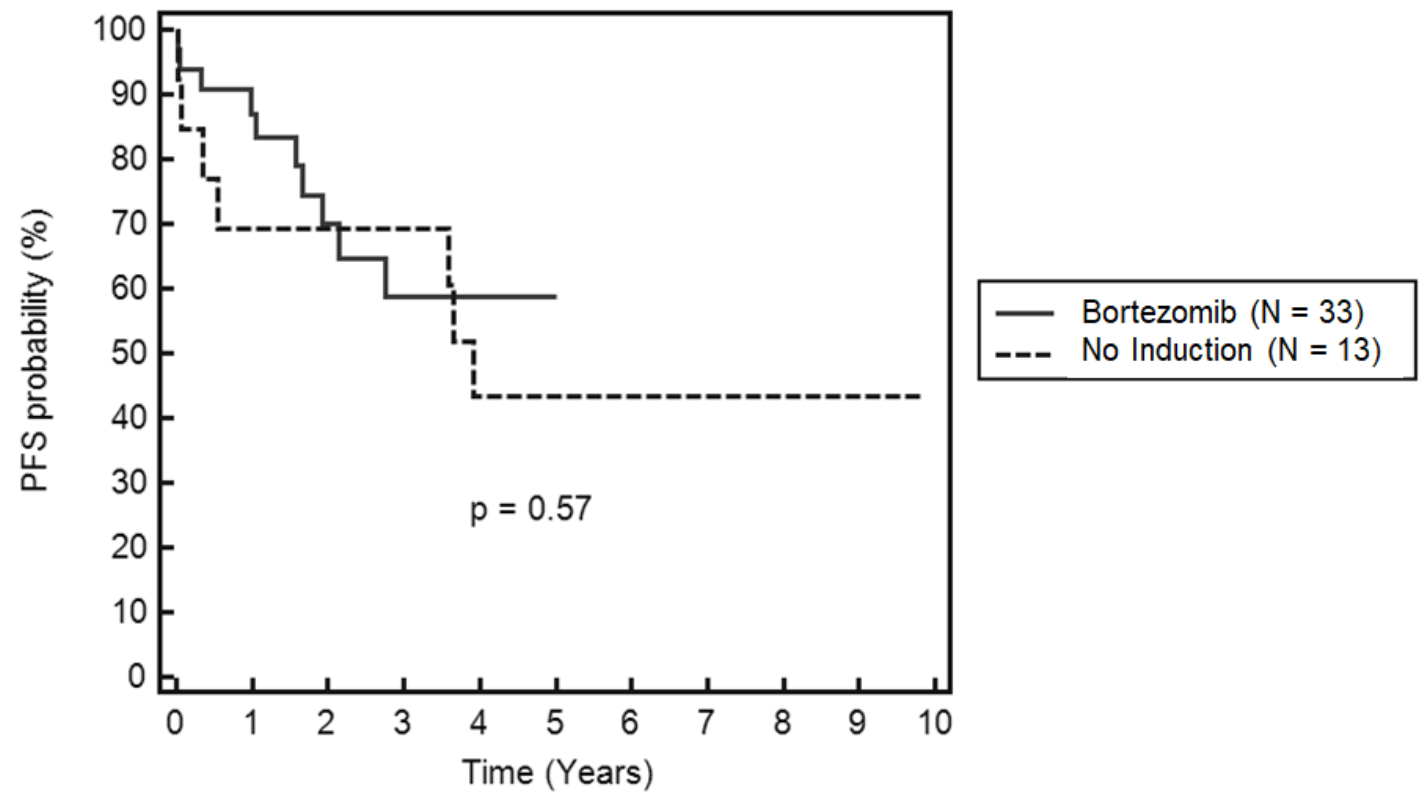

Figure 3. Progression-free survival (PFS) in those receiving bortezomib induction compared to no induction.

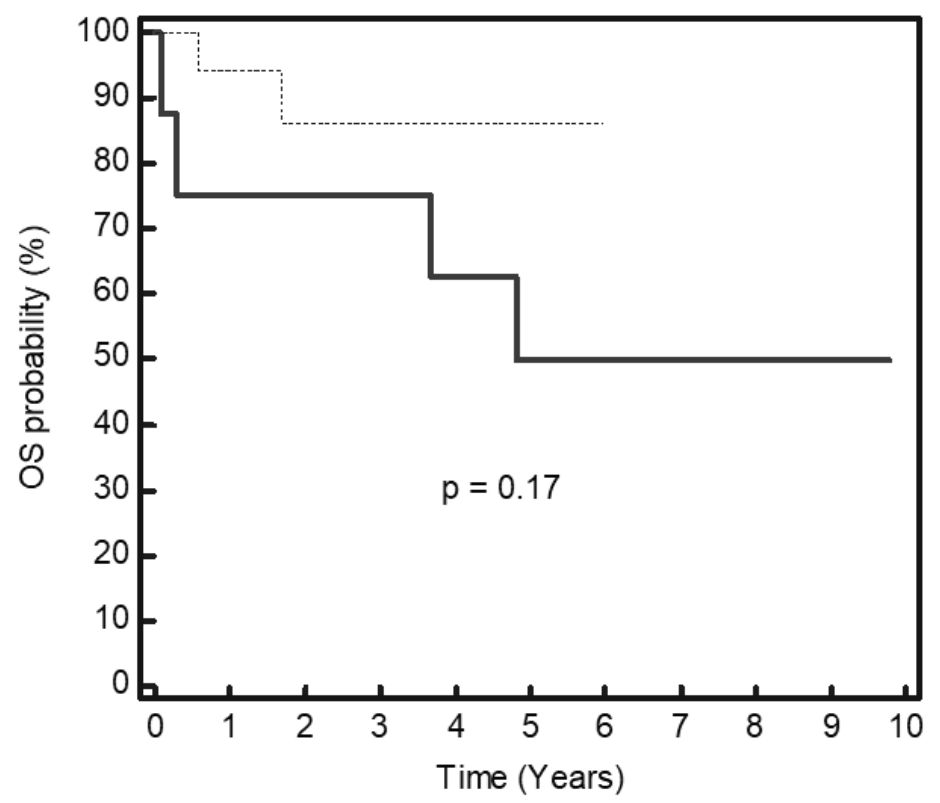

Figure 4. Overall survival (OS) in those with cardiac involvement comparing bortezomib induction to no induction. 


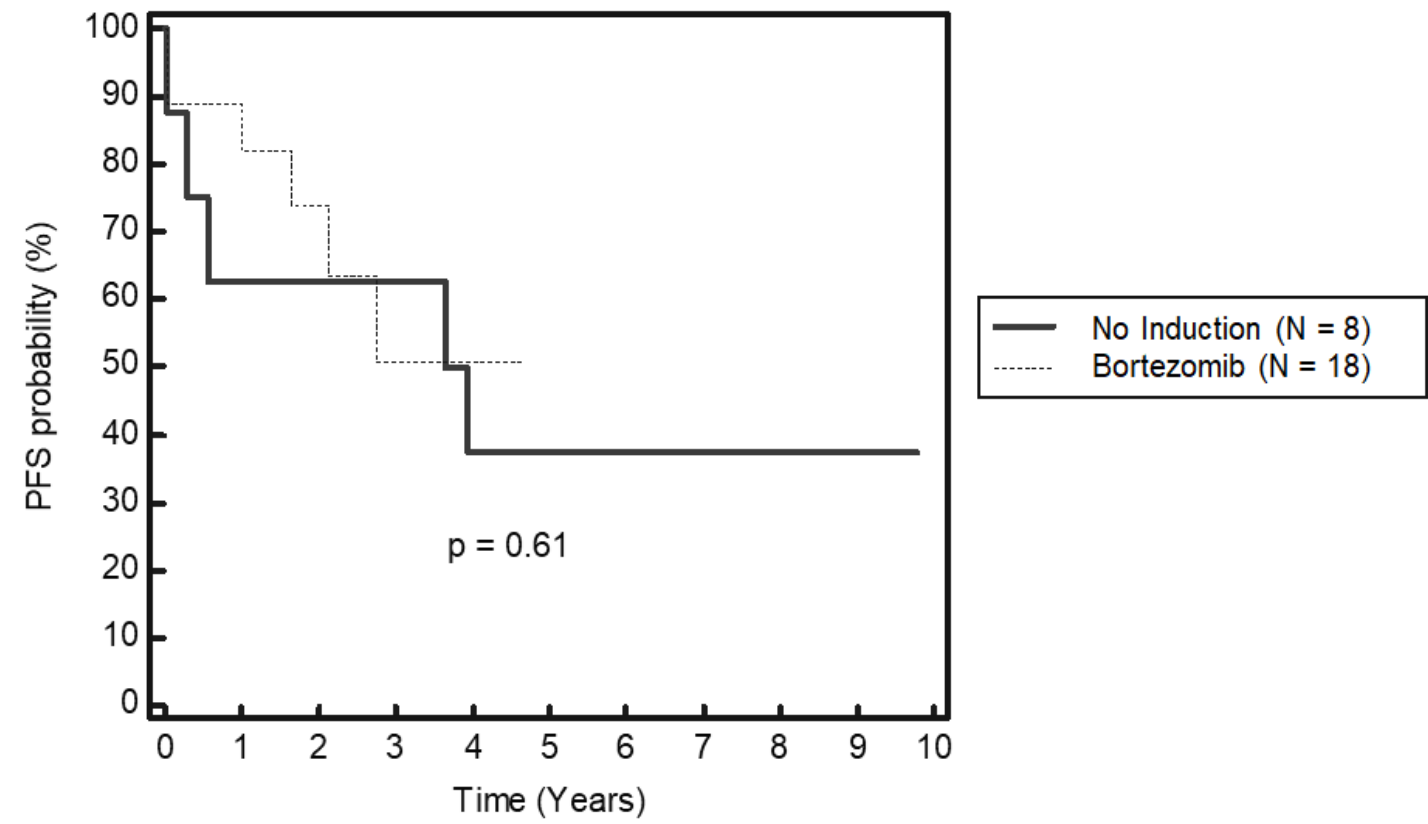

Figure 5. Progression-free survival (PFS) in those with cardiac involvement comparing bortezomib induction to no induction

Two patients died late (greater than 6-months post-ASCT); attribution of cause of death were end stage heart failure and progressive nephrotic syndrome and renal failure.

\section{Discussion:}

Routine use of induction therapy prior to ASCT for patients with AL was still relatively rare at the time of our preliminary analysis, with a retrospective study of a single institution reporting $77 \%$ of patients not receiving induction. ${ }^{18}$ Having shown efficacy in multiple myeloma, ${ }^{19}, 20$ bortezomib-based treatment for $A L$ was receiving consideration for induction, consolidation, and as stand-alone therapy for those ineligible for transplant or with relapsed disease. Specifically, small retrospective analyses of single-agent bortezomib and CyBorD, as well as a prospective analysis of VD induction had shown rapid and high rates of hematologic response. ${ }^{14,21,22}$ Our initial single-institution analysis of 31 patients showed that bortezomib-based induction was well-tolerated and associated with higher hematologic and organ responses compared to noinduction (though comparative analyses for statistical significance were not performed given the small sample size). ${ }^{11}$

Recently, there has been an increasing awareness of the value of bortezomib-based induction for the treatment of systemic AL amyloidosis. However, likely attributed to the rarity of disease and inherent limitations in sample size, data continues to be largely limited to small retrospective and prospective case series. These series have generally yielded favorable results in the setting of planned induction prior to transplant, in patients deemed ineligible for transplant, and in relapsed disease. ${ }^{10,13,23-28}$ 
Additionally, several comparative studies have also reported favorable outcomes with bortezomib-based induction. A 2014 matched case-control study of 174 newly diagnosed patients (many with advanced cardiac involvement) comparing frontline bortezomib/melphalan/dexamethasone (BMDex) with melphalan/dexamethasone (MDex) showed higher rate of CR with BMDex. There was no difference in overall survival between the groups in the overall population, however there was a survival advantage with BMDex in patients with lower-risk cardiac involvement. ${ }^{29}$ Another 2014 matched case-control study by Venner et al comparing 69 patients treated with frontline CyBorD with 69 patients treated with cyclophosphamide/thalidomide/dexamethasone (CTD) showed higher rate of CR and more prolonged PFS with CyBorD, but no difference in 1-year OS, even when stratifying by degree of cardiac involvement. ${ }^{30}$ Sidana et al assessed transplant-ineligible patients and showed higher hematologic response rates (with higher rates of early VGPR at 3 and 6 months) with bortezomib-based induction compared with no-induction, with faster cardiac response and lower relapse rate, but no difference in overall survival. ${ }^{31}$ Most recently, in 2016, Kastritis et al presented the preliminary results of the first phase III randomized trial of 110 patients with untreated AL ineligible for transplant comparing bortezomib/melphalan/dexamethasone (BMDex) vs. melphalan/dexamethasone alone; BMDex showed a more frequent and deeper hematologic response rate, with equivalent renal and cardiac response, but no difference in survival. ${ }^{32}$

Our current analysis continues to support bortezomib-based induction in patients with AL, with significantly higher hematologic response rate and shorter times to maximum hematologic response compared to no induction prior to ASCT. Importantly, with bortezomib pre-ASCT treatment, several patients initially ineligible for transplant ultimately became ASCT-eligible, further supporting the benefits of this therapy in this population, particularly given the excellent longterm survival benefit associated with ASCT alone. ${ }^{2,33}$ A limitation of our study is that it does not include those that initiated bortezomib and did not reach ASCT. An intent-to treat prospective analysis would undoubtedly result in more balanced results, and les biased data. In our subjects, bortezomib induction was well-tolerated, with all cases of early mortality (before day 100 of ASCT) occurring in transplant patients with high disease burden who did not receive pretransplant disease burden reduction. This benefit was seen despite $43 \%$ having cardiac involvement and over $25 \%$ of our population having amyloid involvement of 3 or more organs, both of which are considered by some to be a contraindication to ASCT. No cases of early mortality were observed in our recent cohort, which may be attributed to improvements in peritransplant care in recent years, but also may reflect the improved performance status achieved and the debulking therapy administered. ${ }^{1}$

Although hematologic response rates were more favorable with bortezomib-based induction, the depth of hematologic response, organ response, cardiac and renal response, PFS, and OS did not reach statistical significance, but these results are likely limited by sample size. However as the population matures and responses are sustained, these favorable rates could evolve to statistical significance. Nonetheless, previous studies have shown that attaining hematologic response (even partial response) is associated with high organ response rates and improved survival. ${ }^{34-36}$ Therefore, a primary goal of therapy is to reach hematologic response (an outcome improved with bortezomib-based induction in this study), as the elimination of pathologic monoclonal protein and plasma cell clone may facilitate reversal of organ damage and dysfunction. 
Despite the limitations of our small retrospective analysis, our results appear comparable to other transplant center reports, showing that bortezomib-based induction is well-tolerated and associated with frequent and rapid hematologic responses. These findings therefore continue to support the emerging roles for bortezomib-based induction regimens for the purposes of improving response and even increasing eligibility for patients to safely undergo HDM + ASCT. Larger scale analyses in the form of prospective multi-center trials would be beneficial to further study these roles.

\section{Acknowledgements:}

The authors of this article thank the patients and their families/caregivers, the OHSU multidisciplinary team (consisting of specialists from cardiology, nephrology, and neurology), the hemato- and renal pathology team, and the remainder of our malignant hematology and stem cell transplantation colleagues.

\section{Conflict of interest:}

The authors have no conflicts of interest.

\section{References:}

1. D'Souza A, Dispenzieri A, Wirk B, Zhang MJ, Huang J, Gertz MA et al. Improved Outcomes After Autologous Hematopoietic Cell Transplantation for Light Chain Amyloidosis: A Center for International Blood and Marrow Transplant Research Study. Journal of clinical oncology : official journal of the American Society of Clinical Oncology 2015; 33(32): 3741-3749. e-pub ahead of print 2015/09/16; doi: 10.1200/jco.2015.62.4015

2. Cibeira MT, Sanchorawala V, Seldin DC, Quillen K, Berk JL, Dember LM et al. Outcome of AL amyloidosis after high-dose melphalan and autologous stem cell transplantation: long-term results in a series of 421 patients. Blood 2011; 118(16): 4346-4352. e-pub ahead of print 2011/08/11; doi: 10.1182/blood2011-01-330738

3. Skinner M, Sanchorawala V, Seldin DC, Dember LM, Falk RH, Berk JL et al. High-dose melphalan and autologous stem-cell transplantation in patients with AL amyloidosis: an 8-year study. Annals of internal medicine 2004; 140(2): 85-93. e-pub ahead of print 2004/01/22;

4. Jaccard A, Moreau P, Leblond V, Leleu X, Benboubker L, Hermine $\mathrm{O}$ et al. High-dose melphalan versus melphalan plus dexamethasone for AL amyloidosis. The New England journal of medicine 2007; 357(11): 1083-1093. e-pub ahead of print 2007/09/15; doi: 10.1056/NEJMoa070484

5. Gertz MA, Lacy MQ, Dispenzieri A, Hayman SR, Kumar S. Transplantation for amyloidosis. Current opinion in oncology 2007; 19(2): 136-141. e-pub ahead of print 2007/02/03; doi: $10.1097 /$ CCO.0b013e32801494c6

6. Vesole DH, Perez WS, Akasheh M, Boudreau C, Reece DE, Bredeson CN. High-dose therapy and autologous hematopoietic stem cell transplantation for patients with primary systemic amyloidosis: a Cen- 
Autologous Stem Cell Transplant in the Era of Bortezomib-Based Induction for AL Amyloidosis - A Single Institution 11 Year Experience

ter for International Blood and Marrow Transplant Research Study. Mayo Clinic proceedings 2006; 81(7): 880-888. e-pub ahead of print 2006/07/14; doi: 10.4065/81.7.880

7. Comenzo RL, Vosburgh E, Falk RH, Sanchorawala V, Reisinger J, Dubrey S et al. Dose-intensive melphalan with blood stem-cell support for the treatment of AL (amyloid light-chain) amyloidosis: survival and responses in 25 patients. Blood 1998; 91(10): 3662-3670. e-pub ahead of print 1998/06/20;

8. Gertz MA, Lacy MQ, Dispenzieri A, Hayman SR, Kumar SK, Leung N et al. Effect of hematologic response on outcome of patients undergoing transplantation for primary amyloidosis: importance of achieving a complete response. Haematologica 2007; 92(10): 1415-1418. e-pub ahead of print 2007/09/05;

9. Velcade (bortezomib) [package insert]. Millennium Pharmaceuticals, Inc., Cambridge, MA. 2008.

10. Cornell RF, Zhong X, Arce-Lara C, Atallah E, Blust L, Drobyski WR et al. Bortezomib-based induction for transplant ineligible AL amyloidosis and feasibility of later transplantation. Bone marrow transplantation 2015; 50(7): 914-917. e-pub ahead of print 2015/04/29; doi: 10.1038/bmt.2015.73

11. Scott EC, Heitner SB, Dibb W, Meyers G, Smith SD, Abar F et al. Induction bortezomib in Al amyloidosis followed by high dose melphalan and autologous stem cell transplantation: a single institution retrospective study. Clinical lymphoma, myeloma \& leukemia 2014; 14(5): 424-430.e421. e-pub ahead of print 2014/03/22; doi: 10.1016/j.clml.2014.02.003

12. Chen Al, Bains T, Murray S, Knight R, Shoop K, Bubalo J et al. Clinical experience with a simple algorithm for plerixafor utilization in autologous stem cell mobilization. Bone marrow transplantation 2012; 47(12): 1526-1529. e-pub ahead of print 2012/05/09; doi: 10.1038/bmt.2012.74

13. Sanchorawala V, Brauneis D, Shelton AC, Lo S, Sun F, Sloan JM et al. Induction Therapy with Bortezomib Followed by Bortezomib-High Dose Melphalan and Stem Cell Transplantation for Light Chain Amyloidosis: Results of a Prospective Clinical Trial. Biology of blood and marrow transplantation : journal of the American Society for Blood and Marrow Transplantation 2015; 21(8): 1445-1451. e-pub ahead of print 2015/04/11; doi: 10.1016/j.bbmt.2015.04.001

14. Mikhael JR, Schuster SR, Jimenez-Zepeda VH, Bello N, Spong J, Reeder CB et al. Cyclophosphamidebortezomib-dexamethasone (CyBorD) produces rapid and complete hematologic response in patients with AL amyloidosis. Blood 2012; 119(19): 4391-4394. e-pub ahead of print 2012/02/15; doi: 10.1182/blood-2011-11-390930

15. Gertz MA, Comenzo R, Falk RH, Fermand JP, Hazenberg BP, Hawkins PN et al. Definition of organ involvement and treatment response in immunoglobulin light chain amyloidosis (AL): a consensus opinion from the 10th International Symposium on Amyloid and Amyloidosis, Tours, France, 18-22 April 2004. American journal of hematology 2005; 79(4): 319-328. e-pub ahead of print 2005/07/27; doi: 10.1002/ajh.20381

16. Palladini G, Dispenzieri A, Gertz MA, Kumar S, Wechalekar A, Hawkins PN et al. New criteria for response to treatment in immunoglobulin light chain amyloidosis based on free light chain measurement and cardiac biomarkers: impact on survival outcomes. Journal of clinical oncology : official journal of the American Society of Clinical Oncology 2012; 30(36): 4541-4549. e-pub ahead of print 2012/10/24; doi: 10.1200/jco.2011.37.7614 
Autologous Stem Cell Transplant in the Era of Bortezomib-Based Induction for AL Amyloidosis - A Single Institution 11 Year Experience

17. Comenzo RL, Reece D, Palladini G, Seldin D, Sanchorawala V, Landau H et al. Consensus guidelines for the conduct and reporting of clinical trials in systemic light-chain amyloidosis. Leukemia 2012; 26(11): 2317-2325. e-pub ahead of print 2012/04/06; doi: 10.1038/leu.2012.100

18. Jimenez-Zepeda VH, Franke N, Reece DE, Trudel S, Chen C, Delgado DH et al. Autologous stem cell transplant is an effective therapy for carefully selected patients with AL amyloidosis: experience of a single institution. British journal of haematology 2014; 164(5): 722-728. e-pub ahead of print 2013/11/26; doi: 10.1111/bjh.12673

19. Harousseau JL, Avet-Loiseau H, Attal M, Charbonnel C, Garban F, Hulin C et al. Achievement of at least very good partial response is a simple and robust prognostic factor in patients with multiple myeloma treated with high-dose therapy: long-term analysis of the IFM 99-02 and 99-04 Trials. Journal of clinical oncology : official journal of the American Society of Clinical Oncology 2009; 27(34): 5720-5726. e-pub ahead of print 2009/10/15; doi: 10.1200/jco.2008.21.1060

20. Chanan-Khan AA, Giralt S. Importance of achieving a complete response in multiple myeloma, and the impact of novel agents. Journal of clinical oncology : official journal of the American Society of Clinical Oncology 2010; 28(15): 2612-2624. e-pub ahead of print 2010/04/14; doi: 10.1200/jco.2009.25.4250

21. Reece DE, Hegenbart U, Sanchorawala V, Merlini G, Palladini G, Blade J et al. Efficacy and safety of once-weekly and twice-weekly bortezomib in patients with relapsed systemic AL amyloidosis: results of a phase 1/2 study. Blood 2011; 118(4): 865-873. e-pub ahead of print 2011/05/13; doi: 10.1182/blood2011-02-334227

22. Sanchorawala V, Shelton AC, Brauneis D, Quillen K, Andrea NT, Sloan JM et al. Treatment of AL Amyloidosis with Two Cycles of Induction Therapy with Bortezomib and Dexamethasone Followed by Bortezomib-High Dose Melphalan Conditioning and Autologous Stem Cell Transplantation. Blood 2012; 120(21): 2019.

23. Alahwal H, Song KW, Duggan P, Sutherland HJ, Neri P, Broady R et al. Bortezomib-Containing Regimens for the Treatment of Newly Diagnosed AL Amyloidosis: Experience of Two Centers in Canada. Blood 2016; 128(22): 2131.

24. Kikukawa Y, Yuki H, Hirata S, Ide K, Nakata H, Miyakawa T et al. Combined use of bortezomib, cyclophosphamide, and dexamethasone induces favorable hematological and organ responses in Japanese patients with amyloid light-chain amyloidosis: a single-institution retrospective study. International journal of hematology 2015; 101(2): 133-139. e-pub ahead of print 2014/11/29; doi: 10.1007/s12185014-1705-9

25. Huang X, Wang $Q$, Chen W, Ren G, Liu Z. Bortezomib with dexamethasone as first-line treatment for AL amyloidosis with renal involvement. Amyloid : the international journal of experimental and clinical investigation : the official journal of the International Society of Amyloidosis 2016; 23(1): 51-57. e-pub ahead of print 2016/02/11; doi: 10.3109/13506129.2016.1138939

26. Jimenez-Zepeda VH, Duggan P, Neri P, Bahlis NJ. Bortezomib-Containing Regimens for the Treatment of Newly Diagnosed and Relapsed Amyloid Light Chain Amyloidosis: A Single-Center Experience. Clinical lymphoma, myeloma \& leukemia 2016; 16(6): e79-84. e-pub ahead of print 2016/04/23; doi: 10.1016/j.clml.2016.03.005

27. Gatt ME, Hardan I, Chubar E, Suriu C, Tadmor T, Shevetz $O$ et al. Outcomes of light-chain amyloidosis patients treated with first-line bortezomib: a collaborative retrospective multicenter assessment. Euro- 
Autologous Stem Cell Transplant in the Era of Bortezomib-Based Induction for AL Amyloidosis - A Single Institution 11 Year Experience

pean journal of haematology 2016; 96(2): 136-143. e-pub ahead of print 2015/04/02; doi: 10.1111/ejh.12558

28. Palladini G, Sachchithanantham S, Milani P, Gillmore J, Foli A, Lachmann H et al. A European collaborative study of cyclophosphamide, bortezomib, and dexamethasone in upfront treatment of systemic AL amyloidosis. Blood 2015; 126(5): 612-615. e-pub ahead of print 2015/05/20; doi: 10.1182/blood-201501-620302

29. Palladini G, Milani P, Foli A, Vidus Rosin M, Basset M, Lavatelli F et al. Melphalan and dexamethasone with or without bortezomib in newly diagnosed AL amyloidosis: a matched case-control study on 174 patients. Leukemia 2014; 28(12): 2311-2316. e-pub ahead of print 2014/07/26; doi: 10.1038/leu.2014.227

30. Venner CP, Gillmore JD, Sachchithanantham S, Mahmood S, Lane T, Foard D et al. A matched comparison of cyclophosphamide, bortezomib and dexamethasone (CVD) versus risk-adapted cyclophosphamide, thalidomide and dexamethasone (CTD) in AL amyloidosis. Leukemia 2014; 28(12): 2304-2310. epub ahead of print 2014/07/17; doi: 10.1038/leu.2014.218

31. Sidana S, Tandon N, Dispenzieri A, Gertz MA, Buadi F, Lacy MQ et al. Bortezomib Versus NonBortezomib Based Treatment for Transplant Ineligible Patients with Light Chain Amyloidosis. Blood 2016; 128(22): 3317.

32. Kastritis E, Leleu X, Arnulf B, Zamagni E, Cibeira MT, Kwok F et al. A Randomized Phase III Trial of Melphalan and Dexamethasone (MDex) Versus Bortezomib, Melphalan and Dexamethasone (BMDex) for Untreated Patients with AL Amyloidosis. Blood 2016; 128(22): 646.

33. Gertz MA, Lacy MQ, Dispenzieri A, Kumar SK, Buadi FK, Dingli D et al. Trends in day 100 and 2-year survival after auto-SCT for AL amyloidosis: outcomes before and after 2006. Bone marrow transplantation 2011; 46(7): 970-975. e-pub ahead of print 2010/10/12; doi: 10.1038/bmt.2010.234

34. Leung N, Dispenzieri A, Lacy MQ, Kumar SK, Hayman SR, Fervenza FC et al. Severity of baseline proteinuria predicts renal response in immunoglobulin light chain-associated amyloidosis after autologous stem cell transplantation. Clinical journal of the American Society of Nephrology : CJASN 2007; 2(3): 440-444. e-pub ahead of print 2007/08/19; doi: 10.2215/cjn.02450706

35. Gertz MA, Lacy MQ, Dispenzieri A, Hayman SR, Kumar SK, Dingli D et al. Autologous stem cell transplant for immunoglobulin light chain amyloidosis: a status report. Leukemia \& lymphoma 2010; 51(12): 21812187. e-pub ahead of print 2010/10/21; doi: 10.3109/10428194.2010.524329

36. Lachmann HJ, Gallimore R, Gillmore JD, Carr-Smith HD, Bradwell AR, Pepys MB et al. Outcome in systemic $\mathrm{AL}$ amyloidosis in relation to changes in concentration of circulating free immunoglobulin light chains following chemotherapy. British journal of haematology 2003; 122(1): 78-84. e-pub ahead of print 2003/06/26; 